\title{
Secondary neurological deficits after a conservatively treated U-shaped sacral fracture A Adelved ${ }^{* 1,2}$, A Tötterman ${ }^{3}$, JE Madsen ${ }^{1}$ and O Røise ${ }^{1}$
}

Address: ${ }^{1}$ Orthopaedic Centre, Ullevaal University Hospital, Oslo, Norway, ${ }^{2}$ Orthopaedic Department, Akershus University Hospital, Lørenskog, Norway and ${ }^{3}$ Department of Orthopaedics, Uppsala University Hospital, Uppsala, Sweden

Email: A Adelved* - aron.adelved@medisin.uio.no

* Corresponding author

from Scandinavian Update on Trauma, Resuscitation and Emergency Medicine 2009

Stavanger, Norway. $23-25$ April 2009

Published: 28 August 2009

Scandinavian Journal of Trauma, Resuscitation and Emergency Medicine 2009, I7(Suppl 3):O3 doi:I0.II86/I757-724I-I7-S3-O3

This abstract is available from: http://www.sjtrem.com/content/I7/S3/O3

(c) 2009 Adelved et al; licensee BioMed Central Ltd.

\section{Introduction}

We present a case report of a rare type of sacral fracture who initially had no neurological deficits in the lower extremities, but later on, during fracture healing developed progressive neurological deficits.

\section{Methods}

The patient, a 60-year-old man, was conscious and haemodynamically stable at admission to the hospital after a car accident in $80 \mathrm{~km} / \mathrm{h}$. No neurological deficits were found. CT- scan showed a U-shaped fracture of the sacrum in the S2 level and bilateral fracture of the transverse processes in $\mathrm{L} 5$. The pelvic ring was intact. The fracture was thus treated conservatively, since there was insignificant dislocation and no neurological symptoms.

\section{Results}

At 1-year follow up he presented reduced sphincter tonus, but no micturition problems. He reported pain during sexual intercourse and reduction in muscle strength in both lower extremities and bilateral sensibility reduction in the lower legs and feet were documented. At the 3-year follow up the patient reported intensification of the pain in the lower legs and feet in addition to faecal incontinence. Plain x-ray radiographs showed the fracture healed in a slight kyphosis and a large amount of callus of the lateral masses. CT-scan revealed bony obstruction of the S1 and S2 root canals and ossification between L5 transverse processes and lateral masses of the sacrum bilaterally.

\section{Conclusion}

Our case demonstrates a course that is not reported in the literature, describing late debut and progression of neurological deficits after a conservatively treated lumbo-sacral dissociation. The symptoms are most likely result of the excessive ossification in the fractured sacral foramens, the central canal, and ossification between the L5 transverse processes and the sacral lateral masses. The case demonstrates the importance of close and long term clinical follow up and the need for longitudinal clinical studies to evaluate the natural course of sacral fractures. 